\title{
Does chaos theory have major implications for philosophy of medicine?
}

\section{S Holm}

J Med Ethics: Medical Humanities 2002;28:78-81

In the literature it is sometimes claimed that chaos theory, non-linear dynamics, and the theory of fractals have major implications for philosophy of medicine, especially for our analysis of the concept of disease and the concept of causation. This paper gives a brief introduction to the concepts underlying chaos theory and non-linear dynamics. It is then shown that chaos theory has only very minimal implications for the analysis of the concept of disease and the concept of causation, mainly because the mathematics of chaotic processes entail that these processes are fully deterministic. The practical unpredictability of chaotic processes, caused by their extreme sensitivity to initial conditions, may raise practical problems in diagnosis, prognosis, and treatment, but it raises no major theoretical problems. The relation between chaos theory and the problem of free will is discussed, and it is shown that chaos theory may remove the problem of predictability of decisions, but does not solve the problem of free will. Chaos theory may thus be very important for our understanding of physiological processes, and specific disease entities, without having any major implications for philosophy of medicine.

Professor S Holm, Institute

of Medicine, Law and Bioethics, University of Manchester, Williamson Building, Manchester M13 9PL, UK;

soren.holm@man.ac.uk

Professor Holm is also Professor of Medical Ethics at the Centre for Medical Ethics, University of Oslo, Norway

Accepted 28 June 2002
This, then is my vision of what will happen to our scientific perception of disease during the next century: we shall realise the wisdom of the ancient Aristotelian approach to the study of nature, which means that we shall no longer regard disease as a "mechanical fault in the human machine" but as a disturbed life process. We shall apply the theories of open systems and non-linear dynamics to medical problems, and we shall reach a fuller understanding of the development of disease. ${ }^{1}$

n the literature it is sometimes claimed that complexity theory-that is, for the purpose of this paper deterministic chaos theory, nonlinear dynamics, and fractal processes in biology, has major implications for our analyses of the concept of disease and of the concept of disease causation, and that it therefore has major implications for core topics in the philosophy of medicine. Some of the papers making this claim are relatively modest in the extent of the claim they make, ${ }^{1}$ whereas others see complexity theory as just one part of a new all embracing holistic paradigm..$^{2}$ In a series of four articles in the BMJ in September and October of 2001 complexity theory was at the same time presented as the theory by which we would better understand all kinds of systems, from physical and biological to social and societal, and as a theory which would allow us to move beyond reductionistic and mechanistic models. ${ }^{45-7}$

This paper will try to evaluate these claims critically.

\section{PHILOSOPHICAL IMPORTANCE}

The first step in the analysis is a determination of what would be required for a new scientific theory to have implications for the philosophy of medicine, and more generally what would be required for a new scientific theory to have implications for philosophy.

This is not an easy question to answer because a scientific theory can have implications for philosophy or influence philosophers in a number of ways. The following is not an exhaustive list, but I think that it gives an overview of the most important types of influence. A new scientific theory may:

1. inspire philosophers, for instance by giving them new metaphors to work with

2. challenge implicit empirical or quasi-empirical assumptions in philosophical arguments

3. through its emergence in a scientific field lead to reconceptualisation of ideas in the philosophy of science, for instance about the exact nature of paradigm shifts

4. force a fundamental rethinking of philosophical concepts by showing them to be insufficient to encompass the new insights in science.

It is evident that a new scientific theory may have a major influence on philosophy by inspiring philosophers, without the theory actually forcing a rethinking of any of the basic philosophical concepts. The postulation of a heliocentric instead of a geocentric world system, did, for instance, lead to a paradigm shift in astronomy (and religion) and challenged many philosophers, but it did not force a rethink of philosophical core concepts. On the other hand the move from Aristotelian teleology to Newtonian mechanics in the natural sciences did involve a fundamental rethinking of concepts in philosophy. What I want to argue here is that chaos theory will influence philosophy of medicine by inspiring medical philosophers, but that it will not force us to change any of the basic concepts we are working with. 


\section{A DEFINITION OF CHAOS THEORY}

The current MEDLARS thesaurus defines non-linear dynamics in the following way:

The study of systems which respond disproportionately (non-linearly) to initial conditions or perturbing stimuli. Nonlinear systems may exhibit "chaos" which is classically characterized as sensitive dependence on initial conditions. Chaotic systems, while distinguished from more ordered periodic systems, are not random. When their behavior over time is appropriately displayed (in "phase space"), constraints are evident which are described by "strange attractors". Phase space representations of chaotic systems, or strange attractors, usually reveal fractal self-similarity across time scales. Natural, including biological, systems often display nonlinear dynamics and chaos. ${ }^{8}$

A MEDLINE search from 1998 to the end of 2001 produces 1209 publications with "non-linear dynamics" as a keyword.

As is mentioned in the definition, many biological systems seem to be governed by non-linear dynamics and do exhibit chaotic behaviour in their normal state (in the specific sense of "deterministic chaos"). It has for instance been shown that the interbeat interval of the human heart is chaotic, and that a regular heart beat is a sign of disease and a strong predictor of imminent cardiac arrest. ${ }^{90}$ It has also been shown that many physiological systems are not normally in a stable state of homeostatic balance, but in a dynamically stable state characterised by chaotic fluctuations within a certain area of the phase space. ${ }^{11}$

\section{CHAOS THEORY AND THE DISEASE CONCEPT}

These discoveries show that for certain systems the "healthy" or "normal" state is not characterised by regularity, stability or balance, but by chaos, and this could seem to have implications for our distinction between health and disease because it gives a new conceptualisation of when certain physiological systems are functioning normally and when they are not.

The discoveries made using the new mathematical tools of complexity theory therefore seem to imply new answers to the questions "what is disease?" and "what is health?". The further question we need to answer here is whether these new answers are also philosophically new. Do they add or subtract from the philosophical analysis of the disease concept?

There is no agreement of the proper analysis of the concept of disease in the philosophy of medicine, but it is possible to identify two major schools of thought: a nominalist or normative school, and a realist or functional school. In the next part of the paper I will therefore ask what implication complexity theory has for these two ways of analysing the disease concept.

The nominalist/normative analysis, for instance exemplified by the work of H Tristram Engelhardt Jr, claims that there is an irreducible normative component in claiming that some state of an organism is a state of disease. ${ }^{12}$ There is no value free way of distinguishing between health and disease, and there are no states of an organism which could not justifiably be labelled either health or disease (given some value system). Authors in this school often point to the historical changes in our conceptualisation of certain phenomena which have crossed from being healthy to being disease or vice versa (homosexuality, masturbation, free thinking). On this line of analysis there is thus no "objective fact of the matter" as to whether an organism is healthy or diseased, and it follows straightforwardly that new developments in our understanding of the biological states and developments of organisms (like the findings following from complexity theory) cannot have any bearing on the analysis of the disease concept. What such new developments can do, in cultures that value biological knowledge and classify organismic states in accordance with biological knowledge, is lead to a relabelling of certain states, but such relabelling will still ultimately reflect the underlying value system, and not "biological fact".

On the realist/functional analysis of the concept of disease, classically laid out in the work of Christopher Boorse, disease is an objective state marked by an aberration in the function of the organism. ${ }^{13}$ Other functionalist philosophers also include social functions, or move away from Boorse's statistical analysis of normality, but the basic structure of their analysis is the same. An objective state or function or a goal of the organism is posited and disease is defined as an aberration from the state or function or an inability to reach the goal. The main advantage of a realist/functional analysis of the concept of disease is that it aims at removing value judgments from the ascription of disease. It is thus partly aimed at preventing the abuse created by ascription of disease to persons with divergent sexual orientation, unfashionable political views etc. Whether it succeeds in this aim is highly questionable since most realist/functional analyses contain reference to populations and/or environments, and the necessity to fix the boundaries of populations and the character of environments opens the door for various forms of normativity to creep in.

A further advantage of the realist/functional analysis is that it is in accordance with the common intuition that there are certain biological states that are so aberrant that they "are" diseases, no matter which value system one holds-for example, plague.

Within this school of thought about the disease concept it is clearly the case that complexity theory may lead us to relabel certain states because our new understanding of function in terms of dynamic stability and deterministic chaos has shown that states that we previously took to be normal (under an impoverished view of function, equating function with balance and stability) are really abnormal. It is, however, important to see that this does not in any way affect the basic level of the analysis of the concept. Disease is still aberrant function. We have just replaced one view of function with another, or added yet another way of understanding function to those we already employed earlier. What has changed are the facts, not the basic criteria for deciding which states are healthy and which are diseased.

Our preliminary result so far is thus that complexity theory does not have any interesting philosophical implications for the analysis of the disease concept. This does not, of course, preclude it from having large implications for our knowledge and practice in various branches of medicine.

\section{COMPLEXITY THEORY, CAUSATION, AND DISEASE MODELS}

The other area where complexity theory is often said to have implications for the philosophy of medicine is within our understanding of causation, and thereby in our modelling of pathogenesis. If we go back to the MEDLARS definition of non-linear dynamics we read that non-linear dynamics is:

The study of systems which respond disproportionately (non-linearly) to initial conditions or perturbing stimuli. Non-linear systems may exhibit "chaos" which is classically characterised as sensitive dependence on initial conditions. Chaotic systems, while distinguished from more ordered periodic systems, are not random. When their behaviour over time is appropriately displayed (in "phase space"), constraints are evident which are described by "strange attractors".

The sensitive dependence on initial conditions puts a very naïve conception of causality into serious question-that is, the conception where the effect is believed to be proportional 
to the cause. It also introduces an element of unpredictability in systems which display chaotic behaviour. Even if we know the initial conditions with great precision our ability to predict future states of the system is limited since minute differences in initial conditions may rapidly lead to very different states of the system. In chaotic systems our possibility of accurate prediction therefore deteriorates at a much faster rate than in linear systems, and the states of the system quickly become practically unpredictable. ${ }^{14}$ It is, however, important to distinguish between practical unpredictability and true randomness or non-determination. Within the framework of complexity theory systems may exhibit practical unpredictability, but all their future states are still fully understandable as developments of a deterministic system.

Complexity does not dissolve straightforward physical causality but it can make it much harder to disentangle causal relationships. Let us for instance look at the so called "three body problem" as a classical example of complex behaviour in a physically simple system. One version of this problem deals with the motion of three ideal elastic billiard balls on an ideal frictionless billiard table. The movements of the three balls will be complex - that is, even if we know the velocity, momentum, and direction of movement of each ball at a given time, we have no accurate analytical method of predicting the same measurements at a subsequent time (if the time span is not very short). We also have no methods for deciding exactly why the velocity, momentum, or direction of movement of a given ball has a specific value at a given time. But this does not mean that all of the movements on the table are not caused through perfectly normal physical causal events. The movement of any one of the billiard balls on the table is precisely determined by the specific features of its initial movement and its subsequent collisions with the other balls and the edges of the table - that is, through a long, but complex, chain of simple causal events.

In thinking about disease processes at a general level-that is, at a level higher than the one where we try to model a specific disease, people have often developed what I will call "high level disease models". If we look at high level disease models for individual organisms it is possible to make the following rough classification:

Biological models

- Static

- Functional

Psychosomatic models

Biopsychosocial models

Holistic models

Such high level models may either be intended to explain all disease processes, or the disease processes within a certain subfield of medicine. The value of the models is mainly in their use as heuristics for research, diagnosis, or treatment. For all of these high level models it is characteristic that they posit one or more high level domains-for example, the structure of the organism, psychological coping etc, and then explain disease as a disturbance either within one of these domains or as a disturbance in the interaction between two or more of these domains. They thereby direct the attention of researchers, clinicians, and in some cases politicians, to a consideration of all the domains posited by the given model. They are also used in discussion about the role of various groups of professionals, and in public debates about whether they fulfil their roles satisfactorily. For instance, the common complaint that doctors focus too much on the physical and too little on the psychological or social, implicitly relies on a two or three domain model.

When such models are drawn it is typical that uni or bidirectional arrows are drawn between the various domains or factors of the model in order to indicate the existence, or at least the possibility of uni or bidirectional causal influence between the domains. A psychosomatic model would thus, for instance, posit the (biological) body and the psyche as the two domains of interest, and claim a (usually) bidirectional causal influence between domains.

With regard to high level disease models, complexity theory points to problems with two of the implicit assumptions underlying many models.

The first assumption is that health is a state of functional and organismic stability, where stability is conceptualised in terms of some kind of homeostatic balance.

Complexity theory puts this assumption in doubt on two counts $^{15}$ :

1. The concept of functional stability as balance must, as we have discussed above, be augmented with (and perhaps replaced by) a concept of dynamic functional stability, drawing on concepts from complexity theory.

2. The concepts of organismic stability must be changed towards a more dynamic concept. Organismic stability is often an unargued for, and obviously false, assumption.

The second assumption which is challenged by complexity theory is the assumption that we have a clear understanding of the meaning of the uni and bidirectional arrows in our models, or to be more precise that we have a sufficiently explicated understanding of the concepts of function and causality represented by the arrows. Complexity theory is not going to change our analysis of causality at the most fundamental level—as I have already said, the practical unpredictability which is the consequence of deterministic chaos does not, for instance, eventuate in any form of philosophically important indeterminism-but it may give us good reasons once again to attempt to make our concepts of causality as clear as possible. Incorporating the implications of complexity theory may thus have the effect that our well ordered models become considerably more messy, and the predictions and recommendations we make based on them considerably more tentative. But this is in the end a practical and not a philosophical influence.

\section{CHAOS THEORY AND FREE WILL}

A final area where it is sometimes claimed that chaos theory will change our philosophical concepts and analysis is the area of the very old problem of free will. It is impossible here to give a full account of the discussion about free will, but a simplified account can illustrate the problem and the implications of chaos theory. The problem is simply stated: if the world is fully causally determined, then this must also be true for the events occurring in the human mind, including those leading to our experiences of choosing, of exercising our free will. In this picture it is difficult to find room for true free will-that is, the idea that I do something because I want to do it, not just because some causal chain "makes me do it". ${ }^{16}{ }^{17}$

Here it again becomes important to distinguish whether my decisions that I experience as made with my "free will" are 1) causally determined, or 2) are causally determined and predictable. Complexity theory is one way to introduce unpredictability in a fully causal system, but that does not really save free will. If my decisions are functions of causal, but complex and therefore unpredictable mechanisms, it still does not remove the fact that they are fully causally determined.

Complexity theory does not therefore solve the problem of free will in a deterministic universe, unless what we are worried about is not that our decisions are caused, but that they are predictable.

The attempt to solve the free will problem through complexity theory has some parallels with earlier attempts to solve the problem through the indeterminacy of quantum mechanics and the randomness of some quantum events. If there is true randomness in quantum events, and if quantum events are effective parts of the causal chains leading to decisions, then these decisions are not causally determined and unpredictable. This does, however, not give us the kind of free 
will that we want. Instead of having our decisions determined by inflexible causal chains, we now have them determined by random quantum events. There is still no room for the uncaused, but clearly not random agency that we seem to experience when we make decisions with our free will.

In the same way free will is not saved by being the result of complex causal chains, instead of simple ones. There would still be nothing free about it.

\section{CONCLUSION}

Based on the analysis above the conclusion must be that there is no reason to think that complexity theory will fundamentally and radically change our philosophical understanding and analysis of the concepts of health and disease, but that it may lead us to move certain states from one category to the other. It is also likely that complexity theory will have some impact on the way we develop high level models of disease and health.

It is important to note that complexity theory can be, and often is, used in an inherently reductionistic project in exactly the same way as the non-linear models it aims at replacing. When trying to explain the function (and malfunction) of all kinds of systems (from physical to societal) using one kind of master theory, it really does not matter whether that theory is complex or simple, it is still a reductionistic project.

The major impact of complexity theory is going to be in our low level biological models of specific disease states, as is already evident in the medical literature. How important complexity theory will be in this area is, however, not a philosophical question. It is an empirical question that will be answered in the years to come by biological and medical researchers, who will eventually be able to tell us how many biological systems display medically significant chaotic behaviour.

\section{REFERENCES}

1 Wulff HR. The concept of disease: from Newton back to Aristotle. Lancet 1999;354(suppl):50S.

2 Newman MA. The rhythm of relating in a paradigm of wholeness. Image. Journal of Nursing Scholarship 1999;31:227-30.

3 Copnell B. Synthesis in nursing knowledge: an analysis of two approaches. Journal of Advanced Nursing 1998;27:870-4.

4 Plsek PE, Greenhalgh T. The challenge of complexity in health care. British Medical Journal 2001;323:625-8.

5 Wilson T, Holt T. Complexity and clinical care. British Medical Journal 2001;323:685-8.

6 Plsek PE, Wilson T. Complexity, leadership, and management in healthcare organizations. British Medical Journal 2001;323:746-9.

7 Fraser SW, Greenhalgh T. Coping with complexity: educating for capability. British Medical Journal 2001;323:799-803.

8 Definition downloaded from Medline at PubMed (http:// www.pubmed.gov)

9 Goldberger AL, Rigney DR, West BJ. Chaos and fractals in human physiology. Scientific American 1990;262:42-9.

10 Peng C-K, Mietus J, Hausdorff JM. Long-range anti-correlations and non-Gaussian behavior of the heartbeat. Physical Review Letters 1993;70:1343-6.

11 Goldberger AL. Fractal variability versus pathological periodicity: complexity loss and stereotypy in disease. Perspectives in Biology and Medicine 1997;40:543-61.

12 Engelhardt HT. The foundations of bioethics [2nd ed]. Oxford: Oxford University Press, 1996.

13 Boorse C. On the distinction between disease and illness. Philosophy and Public Affairs 1975;5:49-68.

14 Kaplan DT, Glass L. Understanding nonlinear dynamics. New York: Springer Verlag, 1995.

15 Goodwin BC. How the leopard changed its spots. London: Weidenfeld and Nicholson, 1994.

16 Van Inwagen P. The incompatibility of free will and determinism. Philosophical Studies 1975;27:185-99.

17 Malcolm N. The conceivability of mechanism. The Philosophical Review 1968;77:45-72. 